Laboratorio de Anatomia Descriptiva da Faculàade de, Medicina e Cirurgia de São Paulo, dirigido pelo Prof. Dr. A. Bovero.

\title{
UM .CASO DE RIM EM FERRADURA
}

\author{
OBSERVAÇŌES DE BERNARDO ITAPEMA ALVES \\ E ANTONIO VICENTE DE AZEVEDO.
}

E' riquissima a literatura anatomica, tanto a antiga como a moderna, e de toldos os paizes' no que se nefere á anomalia conhecida pela denominação de rim em ferradura (Hufeisenniere dos allemães; Horse-shoe fusion of kidneys dos inglezes). Existe entre nós uma serie de publicações, não só sobre rinss em ferraldura, mas tambem sobre as anomalivis renaes e ureteraes em geral. Recordamos as interessantes publilcações de M. Couto e Fajardo (1897), de J. Franco (1898); e particularmente as die B. Baptista $(1909,1917,1920)$, e as por elle inspiraldas: de Roqueite Pinito (1911), Bastos d'Avila (1914), e Bittenicourt (1917).

A literatura portugueza é tamibem fertill em publicações sobre este assumpto, contanido-se entre ellas as de Maria Mendesi (1909), Souza Junior (1901), Arruda Furtado (1908), Oliveira Coelho (1910), M. A. de Magalhães (1914), N. Garcia (1914), H. Parreira (1915), as de Pires de Lima (1917) e da sua esicola ('Seaibra e Bastos Moniteiro (1920).

O historico da disposição em esitudo já foi exposto em allgumas das puiblicações acima referiidas, pelo que nos affiastamos completamente desta tarefa; alem disso, a literatura de que dispomos para ım estudo maisi minuicioso deste assumipto é relativamente esicassa, daida a carenicia no niosso meio da maior parte das colleiçõos de jornaes de anatomia onde se acham as fontes bibliographicas.

Miuitas questões e differentes particularildades evvideniciadas na diescripção de carda cası das anomalias renaes conhecidas pelas denominações de rim dystopico, rim em ferradura, rim unico etic. permittiram deduzir vedadeinas classsificacões, todas mais ou menos semelhantes ente si. D'entre as mais relcentes meniclionamos as de Gérard (1905), de Hunitimgton (1907), de Benjaimin Baptista (1909), de Papin (1914).

Pondo de lado os casios de falta congenitia de um rim, com hypertrophia compensatoria do rim remanesicente, que conserva mais ou menos a sua forma normal, com outras anomalias do apparelho uro 
genital (Romiti - 1886 - Hönigsberg - 1902 -, Cutore 1911 -, Ganfini - $1919-$ ), ou sem (B. Baptista e outros); considerando somente as differentes modalijdaldies de fusão ou concrescencia dos rins, os rins em ferradura ou rins em dystopia transversal representam a forma mais frequente deste grupo de anomalias. $\mathrm{Na}$ maioria dos casos os rims apreslentam-se fundidos pellos seus polos inferiores, mais raramente pelos superiores e, ainda de modo verdadeiramente excepcional, pelos dois pollos (rim anmular).

Pode haver tambem concresicenicia em direç̄ão longitudinial, parecendo o pollo inferior de um soldar-sie ao superior do outro ectopico, (end to end ou tandem-fusion - Mc. Murrich - 1898, Hill — 1906, Huntington — 1908, Wilhelmj - 1920).

Os rinis ainda podem ser concrescildos em mlasisa, em bollo, ou en disco, mais ou menos regulares, senido que, nestes casols o rim apparentemenite unico acha-se situaido de um ou die outro lad o da columnia vertebrali, numla das regiões lombares, ou, pelo conitrario, em postição ectopica, deanite da collumna lombar, a nivel do promontorio, ou miesmo na excavação pelvica.

Estas concresscencias podem ser concomitantes ou não á rotação normal dos rins ao longo do eixo longituldilnal, de modo a apresentarem o hilio volitado medial ou venitralmenite.

A multiplicildade das origen e veias renaves nas diffrentes anomallias é quasii absolutamente constanite, bem como a coexistencia freiquente, com os rinis apparente ou realmente unicos ou em ferradura da denominada diuplicidade da veia cava inferior, ou persistencila, com duifferentes modalidades, da veia cardinal posterior esquerda.

Muito pouco consilderado tem sildo o comportamento dos nervos renaes nestes casos de anomalias (Calori - 1888).

O rím em ferradura não somente foi descrilpito na idade adulta e em crianças, mas tambem em embrryões (Hill - 1906; Felix - 1911 — emibryão de 30 mm.; Budlde - 1913 - emibryão de 19 mm.), bem como em outros miammilferos (gato, C. Johnison - 1914).

O interesse das illustracões de cada caso em particular e as deduccões syntheticas que se poldem tirar dahi, não se deve limitar somente á conssideração das varias disposilcões puramente anatomicas encontradas, mas tamber ao valior physiologico dos ongãos modificado, e á importancia cirurgica dos mesmos, tanto mais quanto os orgãos assim aliterados no seu desenvolvimento constituem verdadeiramente loci minoris resistentiae para os proicessos morbidos.

Finalmente não dervemos esıquecer as tentativa de explicação embryologica das diffrentes dispostições comprehendidas na denomina- 
çâo generica de rins dystopicos. Cremos todavia poder affirmar que se a expllicação da geniesie formall é facil de encontrar-se, a genese causal só encontra explilcação por meio de mieras hyportheses.

Passamos, após estas ligeirası considerações syntheticas, á descripcão de um casio por nós enconitradio no curso dios exercicios praticos deste anno. No cadarer n. 1089, de R. R. M. der 29 anmos, brasileiro, branico, natural de Serra Negra, falleesidio na S. Casa de Misericordila desta cidalde de S. Paulo, aos 20 de Jultho die 1921 , com o diagnostico de myelite, e transportado a laboratorio de anatomia da Faculdade de Meddicina, encontramos a disposição de rim em ferradura, semido que nosı foil possivell fazer o diagnosticico desta anomallia após a retirada do jejuno-ileo, tendo sido consiervaldo o. mesenterio. A raiz desite cruzarva uma saliencia transversal, bein visivel, mesmo atravez do peritioneo pariettal, situada um pouco para baixo da reflexão inferior do mesocolion transiverso, e que pela palpação mostrava-se continua com os polos infferiores de cada rim. Fleita a ablação do peritoneo parietal e tomanido-se cuildaido para conservar as relações, pela dissecção cuildaidosa dos vasos arteriosos e venosos prevertebraes com o isolamento systematico dos' seus involucros, foi posta a nú a massa renlal, juntamente com as primeiras vias de excreção da urina.

Tratava-se de facto de dois rins reunildos pellas extremidades infe riores por um isthmo, apresentanido o conjumlcto a forma mais commum de rim em ferraldura, com a conicavidade, volitaida para cima.

Ligeiramiente desilocaldo da sua posição nonmal relał̧ivamenite á columina vertebral e ás costellas, o polo superior do rim direito ch€ga ao nivel do bordo superior da cartilagem de conjugação entre as XI e XII V. L., alcançando a margem inferior da XI costella; o polo do rim esquerdo attinge somenter a margem infferior de XI costellia. diśposição esta, contraria ao que sie dá normalmente, na quali o polo superior do rim esquerdo está situado em um nivel mais abaixo do do rim direito.

O isthmo, achattado no sentildo antero-posterior abraça a cartilagem de conjjugação entre as III e IV V.L., e parte dosı corpos destas duais verteibras. O polo inferior esquerldo desice cerca de 2 $\mathrm{cm}$. mais do que o direito. Comp vemos no esichema junto, o rim esquerdo apresenta uma direção approximadamente vertical, ao passo que o direito é obliquo de cima para baixo e da direita para a esiquerda; esta obliquida de tambem é visivell no isthmo, a ponto de; abstrahindo-se dos sulcos que o limitam na face anterior tem-se a impressão de que foil o rim direito que se alonganido inferiormente transpoz a columna verteibrall, indo se ligar á extemidade correspondente do rim esquerdo. 
Achando-se o rim direito mais affastado lateralmente da collumna vertebrail, por isso mesmo, num planı fronital ellie se acha tamibem mais profunidamente situaldo.

Acresicentamos ao que já foi dito do issthmo que elle tem uma forma irregularmenite quadrilatera, apressentando a face anterior convexa tansversal e longitudinalmente. A face posterior é fortemente concava para traz abraçanldo a columina verteibral e osi vasos prevertebraes. Das duas margens a superior é mais delgada do que a. inferior

A continuidaide do parenchilma renal com as duas porções lateraes que é sem limites nitidosi na faceposterior, é, pelo contrario, na face anterior, proffunldamenite escavada por dolis suilcos', um á direita; outro á esquerda. O sullco que limitia o isththmo á direita sie apresenta em continuação do hilo do rim díreito, e cava na margem inferinr do isthmo uma chanfradura que é limitada á direita por uma formação lobular, que poidemos consilderar como polo infeior do rim direito. Este sulco recelbe por baixo da capsula adiposa a porção inferior da pelvis renal e o inicio do ureter direito.

O sulco á esiquerda, mais largo, mais profundo e vertilical, comprehenideu uma parte do hilo do rim esquerdo, contenido dois calices primarios que se dirigem para cima e para á esquerida. Em correspondencia a este sulico, e por fóra da capsula gordiurosa corre o tronco da arteria mesenterica inferior, senido tão initima sua relação que sú tinha a impressão de que foi o dito tronco que o produziu. Correspondendo ao meio do sulloo a arteria mesenterica inferior dá origem ás arterias collicas suiperior e miedia esquerdas.

$\mathrm{Na}$ face anterior do rilm dieito notamlos o hilo, assemelhando-se a uma fissura longitudinal, obliquamente dirigilda de cima para baixo e um pouco da direita para a esquerda. Esta obliquidaide é menor do que a do eixo longitudinal do rim, de modo que os dois eixos se cruzarn inferiormente. Isto se explica porque a porção cephalica do hilo se inicia sobre a margem medial e a proporção que se torna caudail vae se tornanido anterior, cheganido mesmo na sua parte bem inferior, de onde sae um callice, a se continuar com o sulco direito já descripto.

O polo superior é regularmente arredondado, siendo mais largo e mais espesso do que o homologo do lado opposito.

A margem lateral é regularmente conivexa para fora e para baixo. A medial na sua porção superior, acima da incisura longituddinail, pela qual começa o hilo, é regularmente convexa para dentro; a porção inferior é mais delgada, quasi rectillinea, obliqua para baixo e para a esquerda, continuanido-se com a margem superior do isthmo. 
Sobre a porção medial da face anterior do rim direito repousa a pelvis renal, larga e infumdibuliforme, formaida pella reunião de tres cellices secundarios, resultantes por sua vez da fusão de um maior numero de calices primarios. Ao nivel da parte media do sulco direito, a pelvis, apresentanido um estrangulamento bem evidente ao qual succede logo abaixo u'ma dillatacão fusifforme, continua-se com o ureter.

A porção esquerda do rim em ferradura apresenta-sie distinctamiente lobulada, senldo esta lobulação evidenite tanito sobre a face anterior, quanto nas margens lateral e medial. Assim, vemos na margem lateral duas incisuras uma superior e outra inferior, sendo que esta, muito mais pronunciada, situada na união dos dois terços superıores com o terço inferior, aloja uma arteriola. Tam'bem a margem medial apresenta duas incisura em $_{\mathbb{S}}$ correspondencia aos vesus renaes.

O polo inferior desce um pouco abaixo da margem inferior do isthmo, e é mais largo do que o correspondente no rim direito.

$\mathrm{Na}$ face anterior encontramos suilcos superficiaes matis ou menos em continuação ás fissuras. Em correspondenicia a estes sulcos saem os calices renaies secundarios, em numero de tres; um superior, verticalmente descendente, um medio, posterior á parede da pelvis renal, emergindo do parechima renal logo acima da chanfradura inferior de margem lateral, e finallmente um maior e inferior resulita da reunião de tres grandes calices primarios, dois dos quaes parecem sair do sulco esquerdo, e serem provenientes do isthmo. $O$ bacinete renal resultante é muito pequeno, sendo que os calices renaes superior e inferior irradiam-se segundo ao mesmo plano frontal ao bacinete. Assim constituido, o hilo não tem forma definida, apresentan|do-se discontinuo, não existindo de forma alguma um seio renal, o qual é pello menos esboçado á direita. Do bacinete, sem transição brusca origina-sie o ureter, cujo percurso na sua porção inicial coincide com o eixo longitudinal do rim, apresentanido o parenchima renal uma leve impresisão pela sua passagem.

As arterias e as veiasi são assymetricas e militiplias para amlbos os rins. Para o rim direito dirigem-se tres arterias, uma superior, principal, que originando-se da aorta abdominal ao nivel da I V. L., tem um trajecto quasi horisontal, passando atraz da veia cava inferior, tres centimetros após a sua origem se bifurca em dois ramos, um anterior e outro posterior, os quaes, após eilgumas ramificações penetram pela parte superior do hilo no parenchima renal; uma media, extra-hillar de pequeno callibre, originando-se da aorta 5 cm. abaixo da precedente se dirige para a face positerior do isthmo onde se bifurca num ramo superior, ascendente que penetra no labio 
posterior do hilo, e um ramo inferior que penetra na parede media da face positerior do isthmo; finalimente uma terceira arteriola, inferior, originando-se d'a superficie aniterior da arteria iliaca commum direita, ao nivel da biffurcacão da aorta, com trajecto recurrente, penetra pela parte superior do sullco direito.

Para o rim esquerdo temos quatro ramos arteriaes. Um superior, principal, que se originando da aonta abjominal ao mesmo nivel que a homologa do lado opposito, após ulm trajecto ligeiramiente descendente para a esquerda se bifurca em dois ramos, superior e inferior, os quaes penetram no rim pela parte superior e posterior do calice sulperior; diuas arteriolas medias, originadas isoladamentie da a.orta, altingem o rim ao nivel da chanfradura medial inferior, penetranidio no parenichima renal da face anterior; uma quanta arteriola inferior, poveniente da face. medilal da arteria iliaca commum esquerda, a alguns mim. abaixo lda homolloga do lado opposto, se dirige para a parte inferior dio sullico esquerdo.

Quanto ás velias, nottamos duas para o rim díreito e quatro para 0 esquerido. Da parte superior do hillo direito se constitue um grosso tronco venoso corresipondente á veia renal normall, o qual diriginidose obilquamente para cima, para dentro e para a frente se lança na veia cava inferior. Uma segunda velia, delglada, sae da porção inferior do sullco direito, dirigindo-se para baixo e para denitro, cruzando anteriormente a arterila iliaca com/mlum direfita, vae lançar-se na superficie anterior dia veia iliaca commulm esiquerida, por um tronco commum com uma veia symetrica, proveniente da parte inferior do sullico esquierdo. Allém destra uittimla veniula o rim esquerido possue ainda mais tres, das quaes uma siuperior, vollumosa, correspondente á. veia renal normail, que formamido-se na parte superior do hilo esquerdo decorre obliqulamente para cima e para a direita, recebe no seu percurso uma veia supra renal lançanidio-se após um trajecto de mais ou menos 8 cms. na veia cava inferior. Ulma outra veia accessoria, nascenido no porto de penetração daś duas arteriolas meldias, caminlha a principio enitre ellas, ganha a face posterior da aorta, onde se junta a outra veia proveniente da paredie abdominal posterior formando um itron/co, ao quali se une umla segunida veia accessoria renal, que, originada entre os callices superior e medio ho rim esqueridio, cruza a margem medial deste rim entre as duas incisuras. O grosso tronco resullitante da reunião das diuas veias renaes accessorias, e da velia abidominal posterior mede mais ou menos 4 mm. de callibre, e desemboca na veia cava inferior ao nivel da parte a.lta dia II $\mathrm{V}$ L.

Como complemento a esta desicripção accresicentamos que as capsiluas supra-renaes occupavam as posições normaes, sendo conti- 
guas aos polos superiores de ambos os rins, visto como o deslocamento para baixo da massa renal foi insignificicante. Tamberm os uretieres, apesar de resulitarem de pellvis anombalas apresentam um percurso e reilacões perfeitamente nırmaes.

Damos anigumas meldidas, tomadas da preparação do nosso caso:

$\begin{array}{lcccc}\text { Distancia entre os dois poilos superiores } & \text { cmis. } & 8,1 \\ \text { Margem convexa } & . & \text { cmis. } & 37 . \\ \text { Margem concava } & & \text { cms. } & 20 .\end{array}$

\section{Rim direito:}

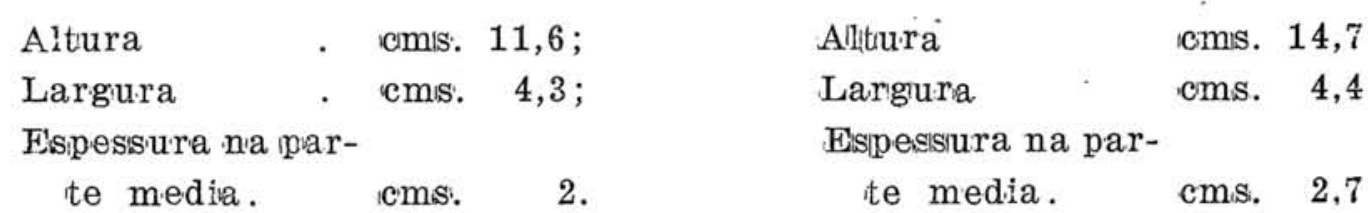

\section{Rim esquerdo:}

Isthmo:

.Espessura

$$
\begin{array}{cc}
\text {. cmis. } & 1,6 \\
\text {. cmis. } & 3,9 \\
\text { cms. } & 3,8
\end{array}
$$

Entendemos: limitar esta nota á rapilda desicripcsão do icaso, no qual não encontramos, de outra piarte, particularidades que posisam parecer novas ou pouco iconheicidas.

A massa renal é ligeiramente desllocada para baixo, porem muito menos do que se observa na maioria dos casos deste grupo (Muthmann. 1907); e possue vasios arteriosos e venosos mulitiplos, o que s normall nestas anomalias.

Temos uma assymetria notavel pela conformação e disposição dos dois rins. De facto o rim direito apresenta uma parte do hilo, a superior, em forma de uma fossa em fissura, icorrespondendo á parte de um seio renal normal, emiquanto que este falta na parte inferior, onde os calices se continuam com o parenchima na face anterior. A' esquerda, o seio é completamente ausente, verificandose a continuidade dos calices primarios, exclusivamente na face anterior do rim.

E' facil uma explicação desta assymetria.

A direita o rim simplesmente iniciou, mas não completou a sua rotação, segundo o seu eixo longitudinal, como acontece no seu 
desenvolvimento normal, resultando assim mais uma torsão do que uma verdadeira rotação. A extremidade inferior do hilo, provavelmente porque o polo inferior foi forçado na sua posição primitiva pela icontinuidade com o parenchima renal do lado opposto, está voltado para deante; a superior, pelo contrario, reentrante, constitue um esboço de seio renal, aberto medialmente.

Contrariamente ao que notamos para o rim direito, o hilo esquerdo está inteiramente deslocado sobre a face anterior, de modo que podemos affirmar que neste rim não houve a minima rotação ao longo do eixo longitudinal.

Esta parada, completa ou incompleta, da rotação normal é muito commum nos rins em ferradura, rins unicos, rins ectopicos, etc. (Watt - 1920)

Merece talvez particular consideração a disposição descripta das veias accessorias á esquerda, e sobre tudo a união da accessoria superior com um tronco venoso parietal proveniente da parede abdominal posterior esquerda e decorrente acima das insersões do musculo psoas desde a IV até a II V. L. Este tronco provavelmente é uma forma rudimentar de persistencia da veia cardinal posterior esquerda. Sobre esta persistencia t'emos um valioso trabalho recentissimo de Benjamim Baptista (1921), persistencia essa támbem commum com maior ou menor evidencia nos casos de alteração do desenvolvimento da parte proximal dos apparelhos urinario e genital.

A explicação mais provavel quanto á genese formal da anomalia por nós descripta, é que a fusão dos dois polos caudaes se deu num periodo no qual dois esboços renaes são muito approximados, principalmente pelos polos inferiores. $O$ tecido mesodermico entre os metanephros desapparece ou não realisa o seu desenvolvimento normal. Por motivos que não estão ainda determinados os polos inferiores dos rins acham-se muito precocemente em icondições de se fundirem.

Esta fusão explica, como já deixamos entrever, a parada successiva da rotação ao longo do eixo longitudinal (Huntigton, Mc. Murrich. Bastos D'Avila e outros).

Finalmente mereceria uma particular menção o papel pathogenetico e o valor pratico que pode apresentar o rim em ferradura, mas para não sahir dos limites a que nos cingimos, e sabendo que um nosso distincto collega, M. Galvão está tratando desse argumento, como assumpto de these, deixamos completamente de lado esta parte.

São Paulo, 15 de outubro de 1921. 


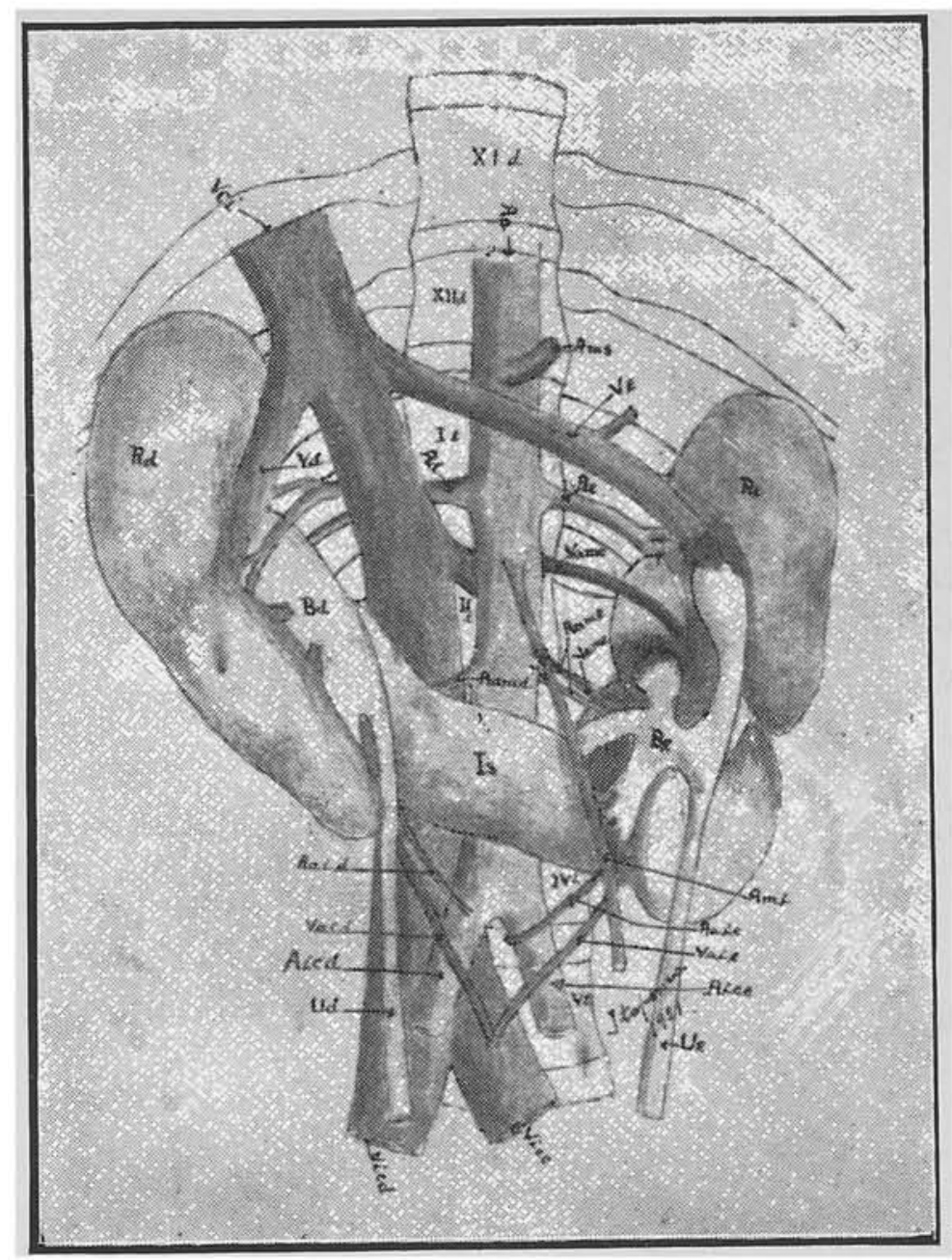



XI D. - XII D. - $11^{\mathrm{a}}$ e $12^{\mathrm{a}}$ vertebras dorsaes.

I L. - II L. - IV L. e V L. - $1^{\mathrm{a}}, 2^{\mathrm{a}}, 4^{\mathrm{a}}$ e $5^{\mathrm{a}}$ vertebras lombares.

Rd. - rim direito; Re. - rim esquerdo; Bd. - bacinete direito; Be. - bacinete esquerdo; Is. - isthmo renal; Vci. - veia cava inferior; Vd. — veia renal principal direita; Ve. — veia renal principal esquerda; Vame. - veias accessorias mediaes esquerdas (reunindo-se posteriormente á aorta com um tronco venoso parietal representante da veia cardinal posterior esquerda); Vaid. - veia renal accessoria inferior direnta; Vaie. - veia renal accessoria inferior esquerda; Vied. — veia iliaca commum direita; Vice. - veia iliaca commum esquerda; Aa. - arteria aorta abdominal; Ams. arteria mesenterica superior; Ad. - arteria renal principal direita; Ae. - arteria renal principal renal esquerda; Aamd. - arteria accessoria medial direnta; Aame. - artreias accessorias mediaes esquerdas; Aaid. - arteria accessoria inferior direita; Aaie. arteria accessoria inferior esquerda; Aied. - arteria iliaca commum direita; Aice. - arteria iliaca commum esquerda; Ami. arteria mesenterica inferior; Ud. - ureter direito; Ue. - ureter esquerdo. 